\title{
Molecular Consequences of Cardiac Valve Development as a Result of Altered Hemodynamics
}

Vinal Menon $^{1}$, Lorain Junor ${ }^{2}$, John Eberth ${ }^{1,6}$, Stephanie Ford ${ }^{3}$, Matt McPheeters ${ }^{4}$, Michael Jenkins ${ }^{4,5}$, Marwa Belhaj ${ }^{1}$ and Jay Potts ${ }^{1,6}$

1. Department of Cell Biology and Anatomy, University of South Carolina School of Medicine, Columbia, USA.

2. Instrumentation Resource Facility, University of South Carolina School of Medicine, Columbia, USA.

3. Division of Neonatology, University Hospitals, Rainbow Babies and Children's Hospital, Cleveland, USA.

4. Department of Biomedical Engineering, Case Western Reserve University, Cleveland, USA.

5. Department of Pediatrics, Case Western Reserve University, Cleveland, USA.

6. Biomedical Engineering Program, College of Engineering and Computing, University of South Carolina, Columbia, USA.

Inlet and outlet valves are formed early during embryogenesis. Abnormally formed valves are, thus, the most common type of congenital heart defects (CHDs). CHDs affect nearly 40,000 babies annually. Unfortunately, most congenital valvular diseases may be asymptomatic until adulthood and treatment often requires valve replacement surgery. It is therefore necessary to understand the mechanisms involved in heart valve development.

The formation of healthy heart valves is dependent on genetic as well as epigenetic factors. Hemodynamics is an important epigenetic regulator of valvulogenesis. The effects of altering hemodynamic loads through the developing heart on valve development have been well explored using advanced imaging techniques such as OCT. However, there are limited studies focussed on understanding the molecular effects of altering intracardiac hemodynamics. Deciphering these molecular pathways is crucial so as to develop therapeutic targets to treat congenital valve diseases that can manifest not only in babies, but adults as well.

The heart is the first organ to form and function in the embryo. The early tubular heart consists of the inner endocardium surrounded by myocardium. The cardiac jelly (endocardial cushion), a layer of extracellular matrix (ECM), lies between these two layers. This cushion undergoes extensive growth and remodelling to form the mature heart valves. Mesenchymal cells, originating from the endocardium, populate the cardiac jelly by means of epithelial-mesenchymal-transition (EMT) to produce the required ECM components. We have previously utilized a 3D in vitro culture system in our laboratory to show that hemodynamic stimuli, such as shear stress, influence the expression/deposition of fibrous ECM proteins in both atrioventricular (AV) and outflow tract (OFT) cushions [1,2]. Our research group was the first to show that altering hemodynamics (shear stress and blood pressure) through the developing chick embryonic heart (in vivo) has direct cellular and genetic consequences [3]. One important finding was that OFT banding led to a significant decrease in OFT cushion volume, as can be seen in 3D AMIRA reconstructions (figure 1), which could potentially lead to the formation of abnormal OFT valves.

The goal of the present study was to further investigate the molecular effects of perturbed intracardiac blood flow on OFT valve development. Blood flow through the chicken embryonic heart (Hamilton and 
Hamburger stage 16/17) was altered by partial constriction (banding) of the OFT at the OFT/ventricle junction (OVJ) as described previously [4]. Using OCT, we determined the average constriction to be $28 \%$. Control embryos were not subjected to banding. Both groups of embryos were allowed to develop for another $24 \mathrm{~h}$. We then analysed the expression/deposition of crucial ECM proteins in OFT of banded vs. control hearts by performing immunofluorescence (IF) on PFA-fixed, paraffin-embedded sections. For each target, IF was carried on 3 embryos from each group, with 3-4 sections per embryo. Images were obtained by confocal microscopy under identical settings.

OFT cushion from banded hearts had a decreased collagen type I expression relative to that from the controls. Periostin was expressed more in the myocardium of OFT from control hearts while OFT from banded hearts had increased periostin localization in the cushion. IST-9 (fibronectin splice variant expressed in mesenchyme) expression was higher in the cushion of banded OFT compared to the OFT of control hearts. There appears to be increased collagen type I in AV cushions from control hearts compared to those from banded hearts. Periostin and IST-9 expression was much higher in the control myocardium and endocardium compared to these regions in the AV cushions of the banded heart. Furthermore, there seems to be a gap between the AV cushions from the banded heart. We speculate the gap between the two AV cushions to be due to changes in pressure in this region due to OFT banding. One of the on-going studies in the lab is to obtain real time pressure readings at different sites in the developing chick heart.

In conclusion, our study shows that altering hemodynamic conditions through the embryonic chick heart not only has consequences at the transcript level, but also leads to differential ECM protein expression and deposition, which could lead to abnormal valve development and thus disease conditions. This study, thus, reinforces the need to maintain normal hemodynamics during heart valve development [5].

\section{References:}

[1] Tan H et al, Dev Biol. 374 (2013), p. 345-356

[2] Biechler SV et al, Front Physiol. 5 (2014)

[3] Menon V et al, J. Cardiovasc. Dev. Dis. 2 (2015), p. 108-124

[4] Menon V et al, J Vis Exp. (2016)

[5] The authors acknowledge funding from SPARC graduate research grant, Cook Biotech, FirstString Research Inc, NIH 2 P20-RR016434-06, NIH INBRE grant for South Carolina P20GM103499, R01 HL126747

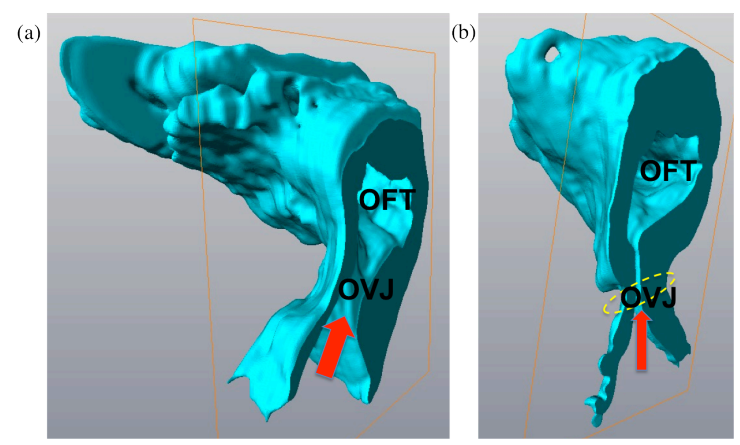

Figure 1. AMIRA 3D reconstruction showing cushion from OFT of (a) control and (b) banded hearts. OFT cushions from banded hearts exhibit a decrease in volume. Arrow indicates direction of blood flow. Yellow dashed line in the banded heart indicates position of suture at the OFT/ventricle junction (OVJ). 